\title{
Hypoxia-induced Long Non-coding RNA LSAMP-AS1 Regulates ceRNA Network to Predict Prognosis for Pancreatic Cancer
}

\section{Lincheng Li}

Medical School of Chinese PLA

Wenbo Zou

Medical School of Chinese PLA

Zhaohui Xiao

Chinese PLA General Hospital, PLA

\section{Zhaoda Deng}

Medical School of Chinese PLA

Rong Liu ( $\sim$ liurong301@126.com )

Chinese PLA General Hospital, PLA

\section{Research Article}

Keywords: hypoxia, TCGA, prognosis, pancreatic cancer, ceRNA

Posted Date: March 1st, 2022

DOI: https://doi.org/10.21203/rs.3.rs-1356897/v1

License: (9) This work is licensed under a Creative Commons Attribution 4.0 International License. Read Full License 


\section{Abstract}

The limited efficacy of chemotherapy and immunotherapy for pancreatic cancer is thought to be largely influenced by the surrounding cancer microenvironment. The hypoxic microenvironment caused by insufficient local blood supply is very important. However, the method to assess the level of hypoxia in the microenvironment of pancreatic cancer (PC) remains unclear. In our research, we downloaded transcriptomic and clinicopathological data from the Cancer Genome Atlas (TCGA) and Gene Expression Omnibus (GEO). A preliminary risk model was developed to reflect the hypoxic environment of pancreatic cancer. We found that high hypoxia risk score indicated poor long-term survival and the presence of an immunosuppressive microenvironment. In addition, based on prognostic hypoxia-related genes, 177 PC samples were divided into two subtypes using a consistent clustering method. Compared with Cluster2, Cluster1 was defined as the "hypoxic subgroup". The infiltration of CD8 T cells, activated memory CD4 T cells, naive B cells, memory B cells, plasma cells, and neutrophils were lower in cluster1, suggesting that there was significant immunosuppression in cluster 1. Then, we constructed a ceRNA regulatory network composed of differentially expressed IncRNA, miRNA and mRNA. Finally, LSAMP-AS1/hsa-miR-129$5 p / S 100 A 2$ has been identified as a key ceRNA network that regulates the hypoxic environment and the prognosis of PC.

\section{Introduction}

Pancreatic cancer (PC) is a fatal cancer with difficult early diagnosis and high mortality, next only to lung cancer and colorectal cancer (1). Most cases of pancreatic cancer are diagnosed in the late stages of the disease, and surgery is not allowed. In addition, due to the poor efficacy of pancreatic cancer radiotherapy, chemotherapy or immunotherapy, the 5 -year survival rate of patients is only $9 \%(2,3)$. Thus, exploring effective biomarkers may be an essential approach for prognosis evaluation and targeted therapy of pancreatic cancer.

In solid tumor tissue, tumor cells mainly rely on tumor blood vessels, oxygen and other nutrients to promote cancer cells invasion and migration (4). During the development of solid tumors, rapidly growing tumors and immature neovascularization lead to the serious deficiency of nutrients and oxygen required by growing tumor cells, resulting in hypoxic microenvironment in cancer (5). According to reports, tumor hypoxia is an important obstacle to cancer radiation, chemotherapy and immunotherapy (6-8). Studying the exact mechanism of hypoxia during the tumor process will create new targets and indicators for cancer treatment and prognosis. However, there are currently no definite indicators of hypoxia in PC.

Non-coding RNAs are extensively involved in the key procession of PC, such as occurrence, development and metastasis, and can predict long-term survival of patients $(9,10)$. Long non-coding RNAs (IncRNAs) are transcriptional RNAs without protein-coding capacity and contain more than 200 nucleotides, and is involved in epigenetic regulation, transcriptional regulation, and post-transcriptional protein-coding (11). MicroRNA binds to the target mRNA response element (MRE) and is the main regulator of posttranscriptional control of gene expression $(12,13)$. The competitive endogenous RNA (ceRNA) hypothesis 
reveals a new mechanism for IncRNA and miRNA. IncRNA competitively bind miRNAs and cover miRNA response elements, thereby reduce the inhibitory effect of miRNA on target mRNA. In addition, IncRNA, as a modulator of mRNA processing, inhibits the expression of mRNA $(14,15)$. The "IncRNA-miRNA-mRNA" network has been validated in a variety of malignancies.

The study identified hypoxia-related genes and establish a prognostic risk model for the hypoxic microenvironment, used consensus aggregation to determine the hypoxic state of $\mathrm{PC}$, and then compared the immune cells infiltration under different hypoxic conditions. Then, the differential expression of IncRNA, miRNA and mRNA related to hypoxia was also analyzed and studied. Based on this, the identification of the key network of hypoxia-related ceRNAs may further improve the tumor regulation mechanism under hypoxia.

\section{Methods}

\subsection{Data Extraction}

We obtained 178 PC samples and 4 normal samples from TCGA (https://www.cancer.gov/tcga). We also downloaded key clinical data such as age, gender, histological grade of tumor, TNM stage, survival time and status. Another dataset, GSE62452 and related clinical data were collected from GEO (https://www.ncbi.nlm.nih.gov/geo/). Somatic mutation data is also obtained from the TCGA database. Gene annotation was performed by Ensembl Genome Database which provided the gtf gene annotation files. Finally, we extracted 200 hypoxia-related genes from Gene Set Enrichment Analysis (hallmarkhypoxia). The expression information of these genes could be obtained from TCGA.

\subsection{Prognostic Model Construction and Verification for Pancreatic Cancer}

To identify hypoxia-related genes related to survival, univariate Cox analysis was used to screen prognostic hypoxia-related genes through the "survival" R package with criteria of $p<0.05$. Prognostic genes are classified as protective or risk genes based on their hazard ratio (HR). The hypoxia-related genes obtained by univariate Cox analysis were included in multivariate Cox analysis. The hypoxiarelated genes with independent prognostic value were identified, and the "survival" and "survminer" $\mathrm{R}$ packages were used to calculate the risk coefficient for each gene. Finally, a proportional hazard regression model determined the risk score formula for calculating the risk score. The structure of the risk assessment formula was as follows:

risk score $=\sum_{i=1}^{N}\left(\operatorname{Exp}_{\mathrm{i}} \times \mathrm{Coe}_{\mathrm{i}}\right)$

Where $\mathrm{N}$ represents the number of genes in the model, $\mathrm{Coe}_{\mathrm{i}}$ represents the corresponding hypoxia gene coefficient, and $\operatorname{Exp}_{\mathrm{i}}$ represents the corresponding hypoxia gene expression level.

Taking the medium risk as the threshold, patients with PC were classified into high-risk and low-risk groups. We draw the overall survival (OS) curve of PC patients in high and low risk groups via the 
"survival" package in R software, and used the "Survival ROC" package to draw ROC curve. We calculated area under the curve (AUC), and evaluated the effect of prognostic model. Then verify the prognostic model through GSE62452.

\subsection{Consensus Clustering for Hypoxia-related Genes}

According to the expression of prognostic hypoxia-related genes, the ConsensuClusterPlus $\mathrm{R}$ package was used to consistently cluster PC samples through unsupervised clustering. The PC samples were divided into two groups of different hypoxic states. The limma package and ggplot2 package were used to analyze and visualize principal component analysis (PCA). Gene set variation analysis (GSVA) was performed to identify the different functional phenotypes that occur under different hypoxic conditions between cluster 1 and cluster 2 using the "GSVA" package. Adjusted P values less than 0.05 was considered statistically significant.

\subsection{Immune Cells Infiltration Analysis}

Using the "CIBERSORT" package, the relative infiltration condition of 22 immune cells in PC was determined. The immune and stromal scores of each PC sample were calculated and evaluated. Then we analyzed the relationship between these cells and hypoxia through the limma package.

\subsection{Differential Expression Analysis and Construction of the ceRNA Network}

The Limma package was performed to analyze the differentially-expressed IncRNA, miRNA, and mRNA between cluster 1 and cluster 2 with false discovery rate $(F D R)<0.05$ and $\mid \log$ foldchange $(F C) \mid \geq 1.5$. Use the miRcode database to study miRNAs that bind to IncRNA, and use three miRNA databases miRDB, miRTarBase and TargetScan to predict miRNA target genes. Once the target relationship of miRNA with mRNA was determined through two of the databases, it was determined as the target gene of the corresponding miRNA. Then the Cytoscape was performed to visualize the IncRNA-miRNA-mRNA regulatory network to better understand the connections. The prognostic values of IncRNA, miRNA and mRNA were analyzed by Kaplan-Meier survival curves.

\subsection{Validation of Crucial IncRNA and mRNA Using qRT-PCR}

The human pancreatic ductal epithelium cell line HPDE6-C7 and pancreatic cancer cell line PANC-1, BxPc3, and SW1990 were purchased from the American Type Culture Collection (ATCC, Manassas, VA). Cells were cultured in high glucose DMEM (Invitrogen, Carlsbad, CA), added with 10\% fetal serum bovine (HyClone, Logan), and placed in a $37^{\circ} \mathrm{C}, 5 \% \mathrm{CO} 2$ incubator. The PC tumors and normal tissues were collected from the Chinese PLA general Hospital. The diagnosis of PC was based on pathological examination. Perform qRT-PCR to check gene expression changes in ceRNA network. In short, use TRIzol reagent (Ambion) to isolate whole RNA from cancer and normal tissues, and use Eppendorf Mastercycler ${ }^{\circledR}$ to convert it into cDNA according to the manufacturer's instructions. The primers were listed in Table 1. 
Table 1

Primers used for qRT-PCR analysis

\begin{tabular}{|lll|}
\hline Gene & Direction & Sequences $\left(\mathbf{5}^{\prime}-\mathbf{3}^{\prime}\right)$ \\
\hline $18 \mathrm{~s}$ & Forward & AACCCGTTGAACCCCATT \\
\hline $18 \mathrm{~s}$ & Reverse & CCATCCAATCGGTAGTAGCG \\
\hline LSAMP-AS1 & Forward & CTGAGCCAGCTTCACTGGAA \\
\hline LSAMP-AS1 & Reverse & TCGTCACATAGGCAGTGTT \\
\hline S100A2 & Forward & GCCAAGAGGGCGACAAGTT \\
\hline S100A2 & Reverse & AGGAAAACAGCATACTCCTGGA \\
\hline
\end{tabular}

\section{Result}

\subsection{Identification of Prognostic hypoxia-related genes in Pancreatic Cancer}

The expression matrix of 200 genes related to hypoxia comes from TCGA RNA-Seq data. With KaplanMeier survival analysis and univariate Cox analysis, we identified 24 genes that are related to hypoxia and are significantly related to the patient's prognosis $(p<0.05)$. Among them, 9 genes have low risk with $\mathrm{HR}<1$, and 15 genes have high risk with $\mathrm{HR}>1$ (Fig. 1A).

\subsection{Construction and Validation of a hypoxia-related genes Prognostic Signature}

To determine the prognosis of PC patients, 24 hypoxia-related genes were used for multiple Cox regression analysis in the TCGA training data set. Eventually, 8 hypoxia-related genes (GCNT2, JMJD6, CA12, STBD1, SIAH2, CAV1, CHST2, and DTNA) were deemed as the powerful prognostic markers and selected to create a prediction model (Fig. 1B). The risk score of each PC sample was calculated as follows: risk score $=(0.49 \times$ GCNT2 $)+(-1.00 \times \mathrm{JMJD} 6)+(0.28 \times \mathrm{CA} 12)+(-0.68 \times \mathrm{STBD} 1)+(1.04 \times \mathrm{SIH} 2)$ $+(0.37 \times \mathrm{CAV} 1)+(-0.68 \times \mathrm{CHST} 2)+(-0.45 \times \mathrm{DTNA})$. Since hypoxia usually leads to a more aggressive cancer phenotype, the prognostic value of the model was investigated. Taking the medium risk score as the cut-off value, the samples were divided into high-risk and low-risk groups, and construct a K-M survival curve based on risk grouping. As shown in Figs. 1C-D, a high hypoxia score in the TCGA cohort was associated with a poorer OS, which was further verified by the GSE62452 cohort $(p<0.05)$. Figures 2A-B shows the distribution of prognostic model, the survival results of different groups of PC patients, and the expression profiles of hypoxia-related genes in the TCGA and GSE62452 databases. Then we calculated the AUC value to evaluate the sensitivity and specificity of the risk score for predicting prognosis of PC patients, the AUC at $1-, 3$-, and 5 - years was $0.761,0.788$, and 0.896 , respectively (Fig. 2C), indicating that the risk model was considerably reliable in predicting the OS of the PC. And that was further validated by GSE62452 datasets (Fig. 2D).

\subsection{Evaluation of $\mathbf{8}$ hypoxia-related gene Signatures as an Independent Prognostic Factor}


Then, to investigate whether the prognostic significance of the signature was independent of other clinicopathological factors, univariate and multivariate Cox regression analysis were performed. The hazard ratio $(\mathrm{HR})$ value and $95 \% \mathrm{Cl}$ of univariate Cox regression analysis were 1.518 and $1.375-1.677(\mathrm{P}$ $<0.001)$, and that of multivariate Cox regression analysis was 1.482 and $1.337-1.643(P<0.001)$ (Table 2), indicted that 8 hypoxia-related genes signature is an independent prognostic factors for PC patients.

Table 2

Univariate Cox regression analysis and multivariate Cox regression analysis for clinical factors

\begin{tabular}{|lllllll|}
\hline Variables & \multicolumn{3}{l}{ univariate Cox } & \multicolumn{4}{l|}{ multivariate Cox } \\
\cline { 2 - 7 } & HR & $\mathbf{9 5 \%}$ IC of HR & P-value & HR & 95\% IC of HR & P-value \\
\hline Sex & 0.893 & $0.591-1.348$ & 0.590 & & & \\
\hline Age & 1.027 & $1.006-1.048$ & 0.013 & 1.015 & $0.994-1.036$ & 0.162 \\
\hline Grade & 1.383 & $1.035-1.847$ & 0.028 & 1.188 & $0.862-1.637$ & 0.292 \\
\hline Stage & 1.363 & $0.937-1.985$ & 0.106 & & & \\
\hline riskScore & 1.518 & $1.375-1.677$ & 0.000 & 1.482 & $1.337-1.643$ & 0.000 \\
\hline
\end{tabular}

The relationship of risk score with clinicopathologic characteristics was also studied, and it found that high-risk score was associated with lymph node metastasis and low immune scores (Fig. 3A).

Furthermore, there were significant differences associated with risk score in terms of immune score $(\mathrm{P}<$ $0.05)$, pathologic_grade $(P<0.05)$, pathologic_T $(P<0.01)$ and pathologic_N $(P<0.05)$ (Supplementary Fig. 1). The risk model we developed is applicable to different ages, genders, degrees, $T$ and $N$ stages (Supplementary Fig. 2).

\subsection{Establishment and Verification of Nomogram}

A prognostic nomogram was successfully developed to predict $1 / 3 / 5$ years survival rate. Age, gender, grade, stage and 8 hypoxia-related gene signatures were included in the final OS prediction model (Fig. 3B). The calibration curve of the nomogram model showed that the predicted overall survival rate of $1 / 3 / 5$ years was well in line with the actual observation results, indicating that the nomogram model has obvious reliability in judging the prognosis of patients with pancreatic cancer (Figs. 3C-E).

\subsection{Characteristics of Immune Cell Infiltration and Tumor Mutation Burden (TMB)}

Then we explored the correlation between prognostic model and immune cell infiltration. According to pearson correlation analysis, the infiltration of naive B cells (cor $=-0.26, p=0.0051)$, Eosinophils (cor $=$ $0.25, p=0.0075)$, CD8 T cells (cor $=-0.24, p=0.01), M 0$ macrophages (cor $=0.26, p=0.0053), M 1$ macrophages (cor $=0.19, p=0.045)$, and activated memory CD4 T cells (cor $=-0.19, p=0.04$ ) was 
significantly correlated with the signature score (Fig. 4A). Then, using the maftools package to analyze the differences in the distribution of somatic mutations between the high-risk and low-risk TCGA cohorts. Figures 4B-C shows that the TMB of the high-risk group is higher than that of the low-risk group (89.74\% vs $65.62 \%)$. TMB has been shown to be a biomarker predicting the clinical benefit of immune checkpoint inhibitor (ICI) treatment. Therefore, high-risk patients are more likely to receive PD-1/PD-L1 immunotherapy.

\subsection{Identification of Potential Hypoxia Subtypes of Pancreatic Cancer}

Based on the expression of 24 genes related to hypoxia, consensus clustering methods were used to cluster the 177 PAAD samples to determine the hypoxia status. The CDF curve has the flattest middle segment, and the interference between subgroups can be minimized when $K=2$. So we chose $K=2$ for consensus cluster analysis to identify two subgroups, cluster $1(n=80)$ and cluster $2(n=97)$ (Fig. $5 A)$, and PCA further indicated that there are significant differences between the two subgroups (Fig. 5B). Besides, we analyzed the prognostic relationship between the two groups and found that the survival rate of cluster2 was higher than that of cluster $1(p<0.001)$ (Fig. $5 C)$. Therefore, compared with cluster 2 , we define cluster 1 as the "hypoxic subgroup". As shown in Fig. 5D, almost all patients with cluster1 subtypes are classified as high-risk groups, which tended to have poorer survival.

Then, according to the distribution of immune cell infiltration, we found that cluster 2 was dominated by naive B cells, memory B cells, plasma cells, CD8 T cells, activated memory CD4 T cells and neutrophils, but only M0 macrophages was upregulated in cluster1 (Fig. 6A). The immune and stromal score were calculated according to the ESTIMATE algorithm, which showed that cluster2 has a higher immune score and stromal score compared with cluster 1 ( $p<0.01$, Fig. 6B). In summary, these results indicate that cluster 2 had more immune cell infiltration. GSVA showed that cluster 1 was rich in cancer-related pathways: Notch signaling pathway, P53 signaling pathway, Glycolysis gluconeogenesis, etc (Fig. 6C).

\subsection{Differentially-Expressed Analysis and Enrichment Analysis of MicroRNAs}

The miRNA mature strand expression RNA-Seq was analyzed to determine the differentially expressed miRNAs associated with hypoxia. A total of 39 differentially-expressed miRNAs were screened with FDR < 0.05 and $|\log F C| \geq 1.5$. Compared with cluster2, there were 3 up-regulated and 36 down-regulated miRNAs in cluster1 (Fig. 7A). In addition, DIANA TOOLS-mirPath v.3 (http://snf-515788.vm.okeanos.grnet.gr/) was used to analyze the KEGG pathway enrichment in these miRNAs. Enrichment analysis results showed that differentially-expressed miRNA is significantly enriched in carcinogenic and hypoxia activation pathways: Proteoglycans in cancer, Focal adhesion, Hippo signaling pathway, HIF-1 signaling pathway, TGF-beta signaling pathway, etc.(Fig. 7B).

\subsection{Establishment of a Competitive Endogenous RNA (ceRNA) Regulation Network associated with Hypoxia}


We analyzed the transcriptomic data of PC to identify hypoxia-related and differentially-expressed genes, including IncRNA and mRNA. To identify whether any IncRNAs might regulate differentially expressed miRNAs, we first used the miRcode database to predict the targeting relationships between differentiallyexpressed miRNAs and IncRNAs, found that the miRNAs, hsa-miR-129-5p, hsa-miR-490-3p and hsa-miR$216 \mathrm{~b}-5 \mathrm{p}$ had negative-related target IncRNAs. Then, the target mRNAs of these three miRNAs were predicted from the three miRNA databases, and a hypoxia-related ceRNA network composed of 5 IncRNAs, 3 miRNAs and 5 mRNAs was developed and visualized by Cytoscape (Fig. 8A). As shown in Figs. 8B-F, among the key genes involved in ceRNA network, LSAMP-AS1, hsa-miR-129-5p, S100A2, HMGA2, and HAVCR1 were significantly correlated with survival $(p<0.05)$. The expression of LSAMP-AS1 was positively correlated with the expression of S100A2 (Fig. 8G). In summary, LSAMP-AS1/hsa-miR-129$5 \mathrm{p} / \mathrm{S} 100 \mathrm{~A} 2$ may be the main ceRNA network that regulates the hypoxic environment and prognosis of pancreatic cancer.

\subsection{Validation of crucial IncRNAs and mRNAs in ceRNA Regulation Network}

qRT-PCR revealed the relative expression of LSAMP-AS1 and S100A2 in PC cells and tissues. As shown in Figs. 9A-D, all these genes were significantly upregulated in $\mathrm{PC}$ cells and tissue compared with normal groups. Further, we also validated the protein expression levels of S100A2 using IHC from the HPA database (https://www.proteinatlas.org/). The protein expression of S100A2 was higher in tumor tissues than in normal tissues (Fig. 10A-B). These results suggested that the LSAMP-AS1/hsa-miR-129$5 \mathrm{p} / \mathrm{S} 100 \mathrm{~A} 2$ axis may be involved in the tumorigenesis of PC.

\section{Discussion}

Pancreatic cancer (PC) is an extremely malignant neoplastic disease with poor prognosis and lack of typical symptoms at an early stage (16). Pancreatic cancer ranks fourth in cancer-related deaths in the United States and is expected to become the second leading cause of death by 2030 (17). Although there have been many advances in pancreatic cancer research in recent years with increasing treatments, the 5year survival rate is still less than $10 \%(18)$. There is an urgent need to study effective molecular biomarkers to establish a theoretical basis for the diagnosis and clinical treatment of PC patients.

Hypoxia is common during cancer progression. The hypoxic microenvironment of tumors is caused by uncontrolled cell proliferation, metabolic changes and vascular abnormalities, which reduce the transport of oxygen and nutrients, leading to tumor progression, metastasis and drug resistance $(19,20)$. PC is usually characterized by severe hypoxia and is closely related to the cancerous process (21). Hypoxia has been confirmed to be an independently risk factor associated with PC prognosis (22). However, the exact mechanism of tumor hypoxic microenvironment remains to be studied. Recent studies have shown that cancer cells can adapt to the hypoxic microenvironment by changing the expression of endogenous genes $(23,24)$.

In our study, we selected 24 hypoxia-related genes from the Kaplan-Meier survival analysis and univariate Cox analysis. Then, the multivariate Cox analysis was used to determine a hypoxia-related risk model 
consisting of eight hypoxia-related genes, including GCNT2, JMJD6, CA12, STBD1, SIAH2, CAV1, CHST2, and DTNA. It has been reported that JMJD6, which is homologous to the hypoxia-inducible factor (HIF) asparaginyl-hydroxylase, can regulate cell proliferation and cause tumorigenesis $(25,26)$. In addition, CA12, a transmembrane protein of the carbonic anhydrase family, was reported to promote tumor growth and metastasis by maintaining intracellular $\mathrm{pH}$ under hypoxia (27). Various studies report that other genes related to hypoxia are largely regulated by the hypoxia response $(28-30)$.

Further evidence shows that hypoxia can prevent the anti-cancer immune response by destroying local immunity and promoting immune suppression (31). According to reports, hypoxia reduces the activity of CD4 + cells, CD8 + T cells and NK cells, and supports immunosuppressive cells such as Treg cells, bone marrow-derived suppressor cells (MDSC) and M2 macrophages (31). Consistent with this, our research shows that the risk score was negatively correlated with naive B cells, CD8 T cells and activated memory CD4 T cells. Besides, the TMB of high-risk patients with pancreatic cancer is statistically higher than the low-risk patients, suggesting that high-risk PC patients may be more sensitive to immunotherapy.

According to the expression of 24 genes related to hypoxia, 177 PAAD patients are divided into two subgroups, cluster 1 and cluster2, to determine the hypoxia state of pancreatic cancer. It has been found that almost all patients with the cluster 1 subtype are classified as high-risk groups, which tended to have poorer survival. Similarly, we analyzed the infiltration of immune cells between cluster1 and cluster2 and found that the infiltration of CD8 + T cells and CD4 + T cells is lower in cluster 1 compared with cluster2, indicating that there may be a degree of immunosuppression in cluster1. This result is consistent with the previous risk model, indicating that severely hypoxic tumor tissue has a higher level of immunosuppression. Gene Set Variation Analysis (GSVA) showed that cluster1 is rich in cancer-related pathways, including Notch signaling pathway, P53 signaling pathway, Glycolysis gluconeogenesis, etc.

Previous studies have demonstrated a significant association between abnormal miRNA expression and hypoxia in various tumors (34-36). Recent studies have reported that hypoxia-induced miRNA upregulation can promote the progression and metastasis of PC by inhibiting the PTEN/AKT pathway. Therefore, miRNAs take a crucial effect in the tumor hypoxic microenvironment. So, we analyzed the differentially-expressed miRNAs in cluster 1 and cluster2 to explore the molecular mechanism of PC progression under hypoxic. KEGG pathway enrichment analysis shows that differentially expressed miRNAs are significantly enriched in carcinogenic and hypoxia activation pathways, including Proteoglycans in cancer, Focal adhesion, Hippo signaling pathway, HIF-1 signaling pathway, TGF-beta signaling pathway, etc.

Further, based on the ceRNA theory, using miRcode, miRBD, miRTarBase and TargetScan database, a hypoxia-related ceRNA network composed of 5 IncRNAs, 3 miRNAs, and 5 mRNAs was constructed. Finally, Kaplan-Meyer's survival analysis found that LSAMP-AS1, hsa-miR-129-5p, S100A2, HMGA2, and HAVCR1 were significantly associated with survival $(p<0.05)$. In summary, LSAMP-AS1/hsa-miR-129$5 p / S 100 A 2$ may be the main ceRNA network that regulates the hypoxic environment and PC prognosis. 


\section{Conclusion}

In summary, we developed a prognostic risk model that used hypoxia-related genes to reflect the hypoxic microenvironment. Notably, we used consensus clustering to determine the hypoxia state in PC, and then compares the immune cell infiltration under different hypoxic conditions. The differential expression of IncRNA, miRNA and mRNA related to hypoxia was also analyzed and studied. On this basis, a core hypoxia-related ceRNA network has been determined, which will further improve the mechanism of tumor regulation during hypoxia. We expect that our research could provide a new perspective on the carcinogenic mechanism and potential targets of pancreatic cancer treatment. But admittedly, our study still has some limitations. We only obtain hypoxia-related genes from the database or previous literature, and the TCGA database does not emerge the true hypoxia level in PC. More functional experiments will be conducted in the future to verify hypotheses and explore further mechanisms.

\section{Declarations}

\section{Author Contributions}

LL, WZ and ZX contributed equally to this work, and were considered as the co-first author. LL participated in design and conception of this study. WZ downloaded the data form corresponding database. ZX performed the bioinformatics analysis in software. ZD contributed to parts of the experiments. All author participated in writing the manuscript. RL proposed the conception and revised the manuscript. All authors read and approved the final manuscript.

\section{Ethics Approval and Consent to Participate}

The ethics committee of PLA General Hospital approved this study and is in compliance with the Helsinki Declaration. Written informed consent was obtained from all individual participants included in the study.

\section{Consent for publication}

Not applicable.

\section{Availability of Data and Materials}

The datasets downloaded for supporting the results of this article are publicly available at the TCGA (https://portal.gdc.cancer.gov/), GEO (https://www.ncbi.nlm.nih.gov/geo/).

\section{Competing interests}


All authors state that there are no potential competing interests.

\section{Funding}

Not applicable.

\section{Acknowledgments}

We acknowledge TCGA and GEO databases for providing their platforms and contributors for uploading their meaningful datasets.

\section{References}

1. Mizrahi JD, Surana R, Valle JW, Shroff RT. Pancreatic cancer. Lancet (2020) 395(10242):2008-20. doi: 10.1016/S0140-6736(20)30974-0.

2. Morrison AH, Byrne KT, Vonderheide RH. Immunotherapy and Prevention of Pancreatic Cancer. Trends Cancer (2018) 4(6):418-28. doi: 10.1016/j.trecan.2018.04.001.

3. Ilic M, Ilic I. Epidemiology of pancreatic cancer. World J Gastroenterol (2016) 22(44):9694-705. doi: 10.3748/wjg.v22.i44.9694.

4. Dhani N, Fyles A, Hedley D, Milosevic M. The clinical significance of hypoxia in human cancers. Semin Nucl Med (2015) 45(2):110-21. doi: 10.1053/j.semnuclmed.2014.11.002.

5. Bhandari V, Hoey C, Liu LY, Lalonde E, Ray J, Livingstone J, et al. Molecular landmarks of tumor hypoxia across cancer types. Nat Genet (2019) 51(2):308-18. doi: 10.1038/s41588-018-0318-2.

6. Teicher BA, Lazo JS, Sartorelli AC. Classification of antineoplastic agents by their selective toxicities toward oxygenated and hypoxic tumor cells. Cancer Res (1981) 41(1):73-81.

7. Graham K, Unger E. Overcoming tumor hypoxia as a barrier to radiotherapy, chemotherapy and immunotherapy in cancer treatment. Int J Nanomedicine (2018) 13:6049-58. doi: 10.2147/IJN.S140462.

8. Hatfield SM, Kjaergaard J, Lukashev D, Schreiber TH, Belikoff B, Abbott R, et al. Immunological mechanisms of the antitumor effects of supplemental oxygenation. Sci Trans/ Med (2015) 7(277):230r-277r. doi: 10.1126/scitranslmed.aaa1260.

9. Zhou C, Yi C, Yi Y, Qin W, Yan Y, Dong X, et al. LncRNA PVT1 promotes gemcitabine resistance of pancreatic cancer via activating Wnt/beta-catenin and autophagy pathway through modulating the miR-619-5p/Pygo2 and miR-619-5p/ATG14 axes. Mol Cancer (2020) 19(1):118. doi: 10.1186/s12943-020-01237-y.

10. Deng SJ, Chen HY, Ye Z, Deng SC, Zhu S, Zeng Z, et al. Hypoxia-induced LncRNA-BX111 promotes metastasis and progression of pancreatic cancer through regulating ZEB1 transcription. Oncogene (2018) 37(44):5811-28. doi: 10.1038/s41388-018-0382-1. 


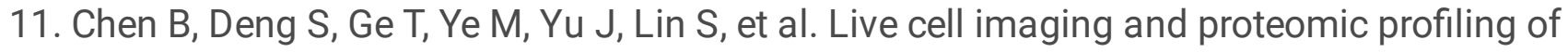
endogenous NEAT1 IncRNA by CRISPR/Cas9-mediated knock-in. Protein Cell (2020) 11(9):641-60. doi: 10.1007/s13238-020-00706-w.

12. Ding JN, Zang YF, Ding YL. MiRNA-146b-5p inhibits the malignant progression of gastric cancer by targeting TRAF6. Eur Rev Med Pharmacol Sci (2020) 24(17):8837-44. doi:

10.26355/eurrev_202009_22823.

13. Pidikova P, Reis R, Herichova I. miRNA Clusters with Down-Regulated Expression in Human Colorectal Cancer and Their Regulation. Int J Mol Sci (2020) 21(13). doi: 10.3390/ijms21134633.

14. Han TS, Hur K, Cho HS, Ban HS. Epigenetic Associations between IncRNA/circRNA and miRNA in Hepatocellular Carcinoma. Cancers (Basel) (2020) 12(9). doi: 10.3390/cancers12092622.

15. Zhang H, Zhu C, He Z, Chen S, Li L, Sun C. LncRNA PSMB8-AS1 contributes to pancreatic cancer progression via modulating miR-382-3p/STAT1/PD-L1 axis. J Exp Clin Cancer Res (2020) 39(1):179. doi: 10.1186/s13046-020-01687-8.

16. Pereira SP, Oldfield L, Ney A, Hart PA, Keane MG, Pandol SJ, et al. Early detection of pancreatic cancer. Lancet Gastroenterol Hepatol (2020) 5(7):698-710. doi: 10.1016/S2468-1253(19)30416-9.

17. Siegel RL, Miller KD, Fuchs HE, Jemal A. Cancer Statistics, 2021. CA Cancer J Clin (2021) 71(1):7-33. doi: 10.3322/caac. 21654 .

18. Allemani C, Matsuda T, Di Carlo V, Harewood R, Matz M, Niksic M, et al. Global surveillance of trends in cancer survival 2000-14 (CONCORD-3): analysis of individual records for 37513025 patients diagnosed with one of 18 cancers from 322 population-based registries in 71 countries. Lancet (2018) 391(10125):1023-75. doi: 10.1016/S0140-6736(17)33326-3.

19. Lu Y, Liu Y, Oeck S, Zhang GJ, Schramm A, Glazer PM. Hypoxia Induces Resistance to EGFR Inhibitors in Lung Cancer Cells via Upregulation of FGFR1 and the MAPK Pathway. Cancer Res (2020) 80(21):4655-67. doi: 10.1158/0008-5472.CAN-20-1192.

20. Wei $X$, Chen $Y$, Jiang $X$, Peng M, Liu Y, Mo Y, et al. Mechanisms of vasculogenic mimicry in hypoxic tumor microenvironments. Mol Cancer(2021) 20(1):7. doi: 10.1186/s12943-020-01288-1.

21. Tao J, Yang G, Zhou W, Qiu J, Chen G, Luo W, et al. Targeting hypoxic tumor microenvironment in pancreatic cancer. J Hematol Oncol (2021) 14(1):14. doi: 10.1186/s13045-020-01030-w.

22. Qin Y, Zhu W, Xu W, Zhang B, Shi S, Ji S, et al. LSD1 sustains pancreatic cancer growth via maintaining HIF1alpha-dependent glycolytic process. Cancer Lett (2014) 347(2):225-32. doi: 10.1016/j.canlet.2014.02.013.

23. de Heer EC, Jalving M, Harris AL. HIFs, angiogenesis, and metabolism: elusive enemies in breast cancer. J Clin Invest (2020) 130(10):5074-87. doi: 10.1172/JCI137552.

24. Yuen VW, Wong CC. Hypoxia-inducible factors and innate immunity in liver cancer. J Clin Invest (2020) 130(10):5052-62. doi: 10.1172/JCl137553.

25. Li L, Wang Q, Yuan Z, Chen A, Liu Z, Wang Z, et al. LncRNA-MALAT1 promotes CPC proliferation and migration in hypoxia by up-regulation of JMJD6 via sponging miR-125. Biochem Biophys Res Commun (2018) 499(3):711-8. doi: 10.1016/j.bbrc.2018.03.216. 
26. Lee YF, Miller LD, Chan XB, Black MA, Pang B, Ong CW, et al. JMJD6 is a driver of cellular proliferation and motility and a marker of poor prognosis in breast cancer. Breast Cancer Res (2012) 14(3):R85. doi: 10.1186/bcr3200.

27. Chen S, Fang XQ, Wang Q, Wang SW, Hu ZJ, Zhou ZJ, et al. PHD/HIF-1 upregulates CA12 to protect against degenerative disc disease: a human sample, in vitro and ex vivo study. Lab Invest (2016) 96(5):561-9. doi: 10.1038/labinvest.2016.32.

28. Mao X, Wong SY, Tse EY, Ko FC, Tey SK, Yeung YS, et al. Mechanisms through Which HypoxiaInduced Caveolin-1 Drives Tumorigenesis and Metastasis in Hepatocellular Carcinoma. Cancer Res (2016) 76(24):7242-53. doi: 10.1158/0008-5472.CAN-16-1031.

29. Nonaka M, Fukuda MN, Gao C, Li Z, Zhang H, Greene MI, et al. Determination of carbohydrate structure recognized by prostate-specific $\mathrm{F} 77$ monoclonal antibody through expression analysis of glycosyltransferase genes. J Biol Chem (2014) 289(23):16478-86. doi: 10.1074/jbc.M114.559047.

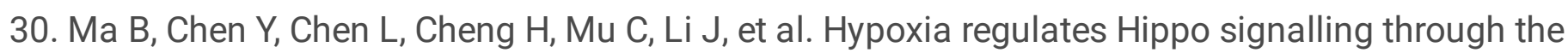
SIAH2 ubiquitin E3 ligase. Nat Cell Biol(2015) 17(1):95-103. doi: 10.1038/ncb3073.

31. Noman MZ, Hasmim M, Messai Y, Terry S, Kieda C, Janji B, et al. Hypoxia: a key player in antitumor immune response. A Review in the Theme: Cellular Responses to Hypoxia. Am J Physiol Cell Physiol (2015) 309(9):C569-79. doi: 10.1152/ajpcell.00207.2015.

32. Multhoff G, Vaupel P. Hypoxia Compromises Anti-Cancer Immune Responses. Adv Exp Med Biol (2020) 1232:131-43. doi: 10.1007/978-3-030-34461-0_18.

33. Damgaci S, Ibrahim-Hashim A, Enriquez-Navas PM, Pilon-Thomas S, Guvenis A, Gillies RJ. Hypoxia and acidosis: immune suppressors and therapeutic targets. Immunology (2018) 154(3):354-62. doi: 10.1111/imm.12917.

34. Ullmann P, Nurmik M, Schmitz M, Rodriguez F, Weiler J, Qureshi-Baig K, et al. Tumor suppressor miR215 counteracts hypoxia-induced colon cancer stem cell activity. Cancer Lett (2019) 450:32-41. doi: 10.1016/j.canlet.2019.02.030.

35. Gervin E, Shin B, Opperman R, Cullen M, Feser R, Maiti S, et al. Chemically Induced Hypoxia Enhances miRNA Functions in Breast Cancer. Cancers (Basel) (2020) 12(8). doi: 10.3390/cancers12082008.

36. Xu K, Zhan Y, Yuan Z, Qiu Y, Wang H, Fan G, et al. Hypoxia Induces Drug Resistance in Colorectal Cancer through the HIF-1alpha/miR-338-5p/IL-6 Feedback Loop. Mol Ther(2019) 27(10):1810-24. doi: 10.1016/j.ymthe.2019.05.017.

37. Cao W, Zeng Z, He Z, Lei S. Hypoxic pancreatic stellate cell-derived exosomal mirnas promote proliferation and invasion of pancreatic cancer through the PTEN/AKT pathway. Aging (Albany NY) (2021) 13(5):7120-32. doi: 10.18632/aging.202569.

\section{Figures}


A

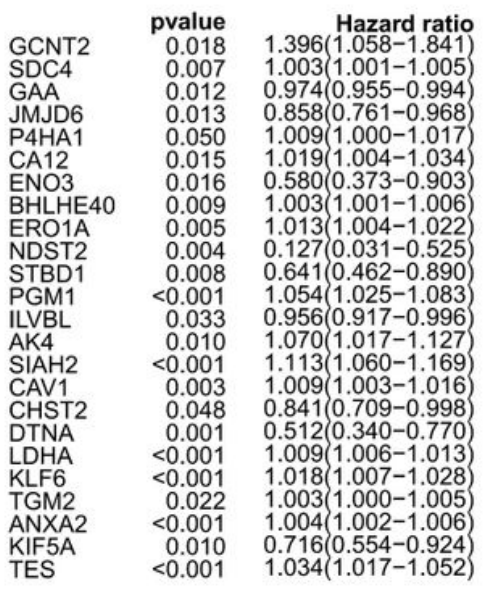

B

$\begin{array}{lrr} & \text { pvalue } & \text { Hazard ratio } \\ \text { GCNT2 } & 0.029 & 1.631(1.050-2.533) \\ \text { JMJD6 } & 0.001 & 0.368(0.201-0.674) \\ \text { CA12 } & 0.004 & 1.321(1.091-1.600) \\ \text { STBD1 } & 0.119 & 0.506(0.215-1.192) \\ \text { SIAH2 } & 0.005 & 2.837(1.378-5.839) \\ \text { CAV1 } & 0.002 & 1.453(1.146-1.843) \\ \text { CHST2 } & 0.012 & 0.505(0.297-0.859) \\ \text { DTNA } & 0.149 & 0.640(0.349-1.173)\end{array}$
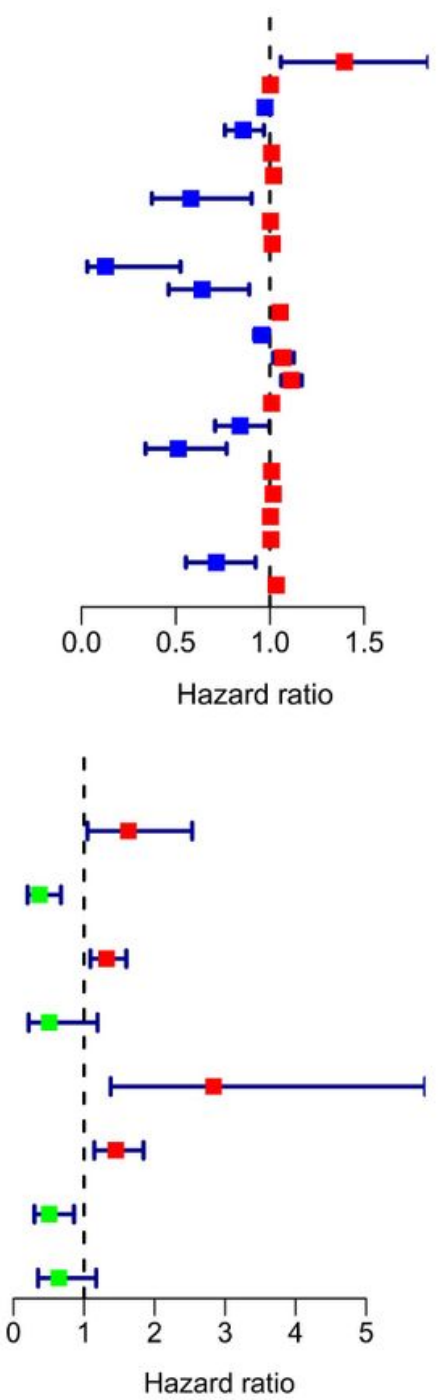

$\mathrm{C}$
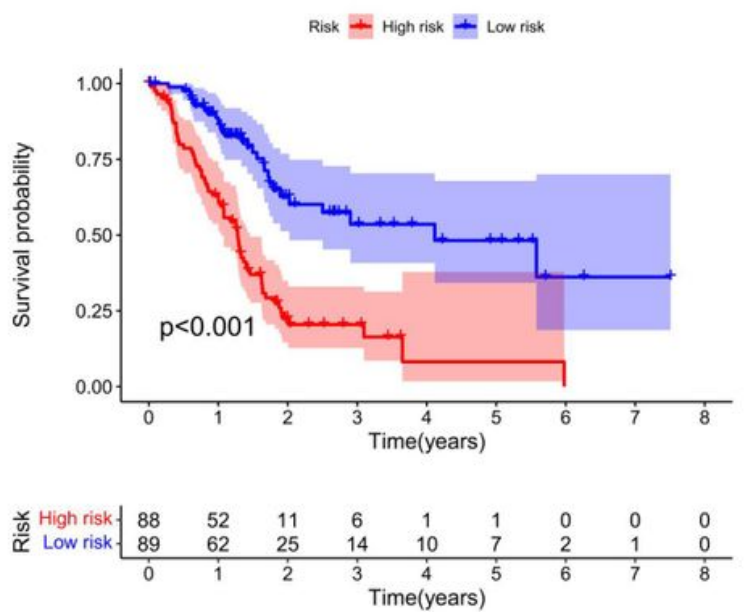

$\mathrm{D}$

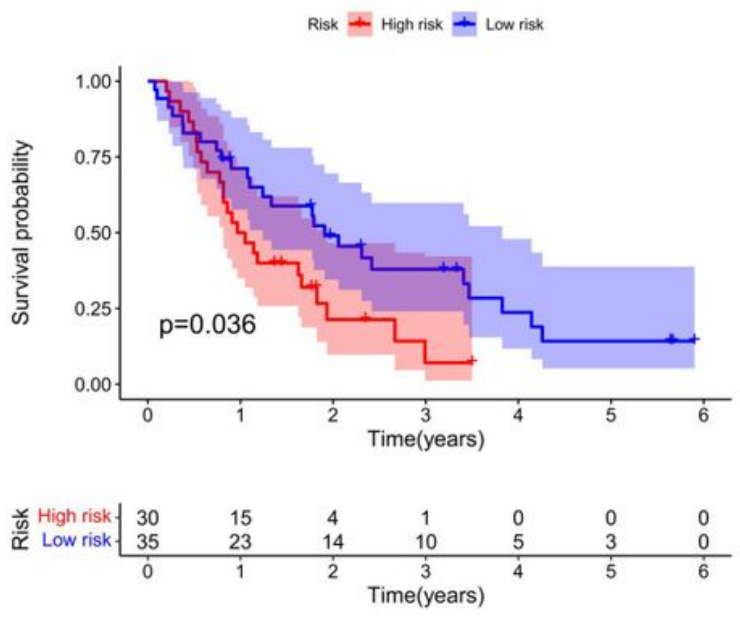

Figure 1

Construction and validation of a hypoxia-related genes prognostic signature. (A) The univariate Cox regression analysis and (B) multivariate Cox regression analysis for hypoxia-related genes in pancreatic cancer. (C-D) Kaplan-Meier survival analysis of high-risk and low-risk risk group in the TCGA cohort and GSE62452 cohort. 
A
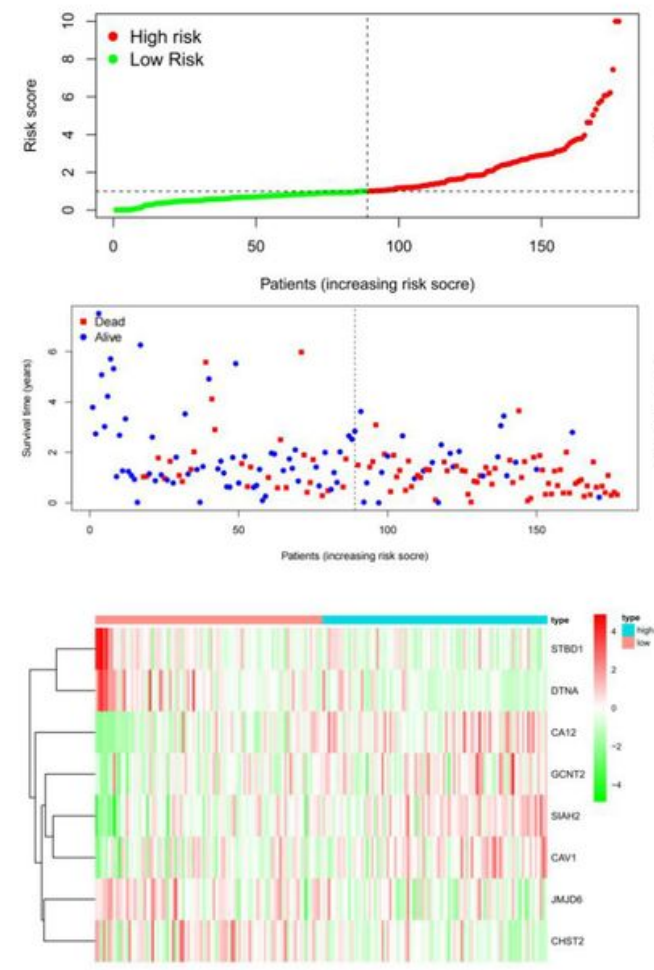

B
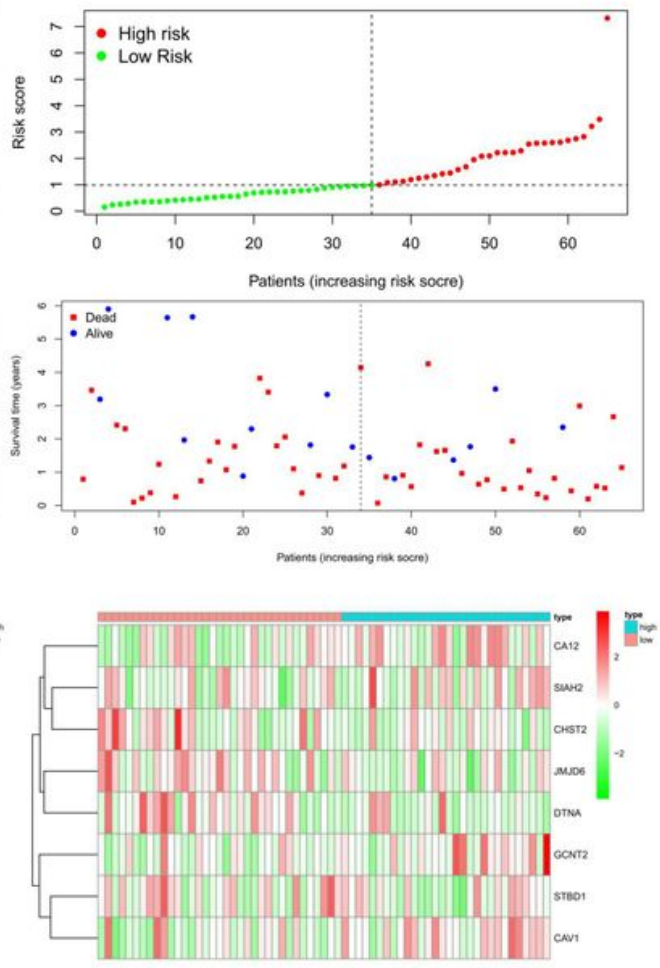

C

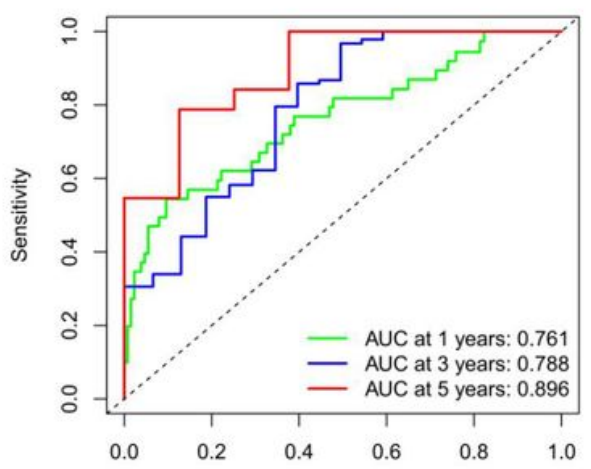

D

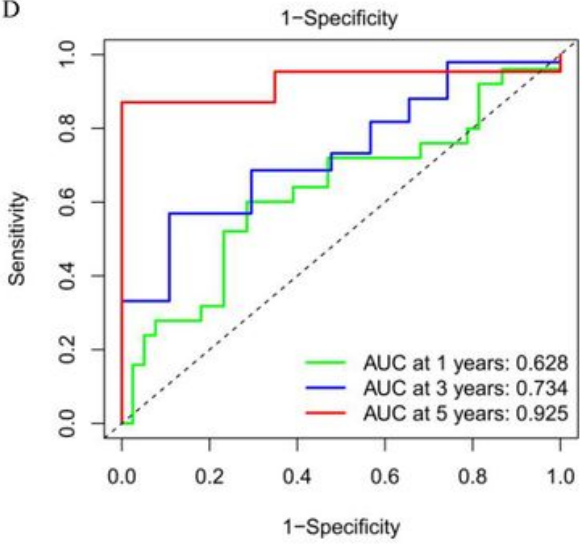

Figure 2

Evaluation of the performance of the eight gene signature in TCGA and GSE62452 datasets. (A-B) The distribution of risk score, survival time and gene expression in the TCGA cohort and GSE62452 cohort. (CD) Receiver operating characteristic (ROC) curves for 1,3, and 5 years survival in both cohort. 
A

A

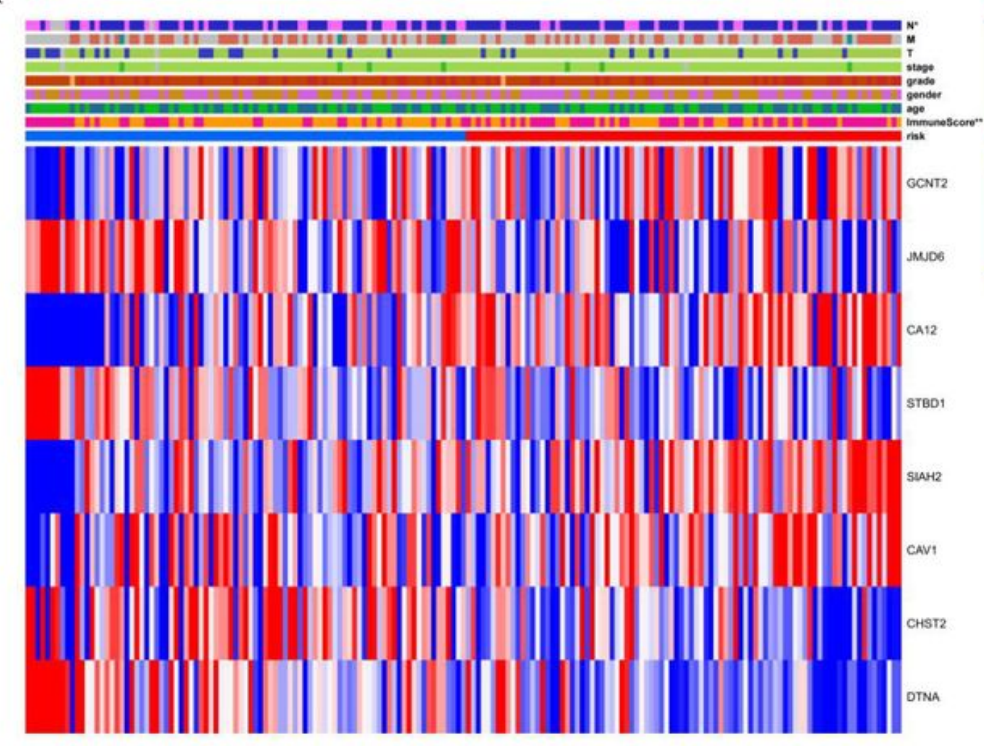

C

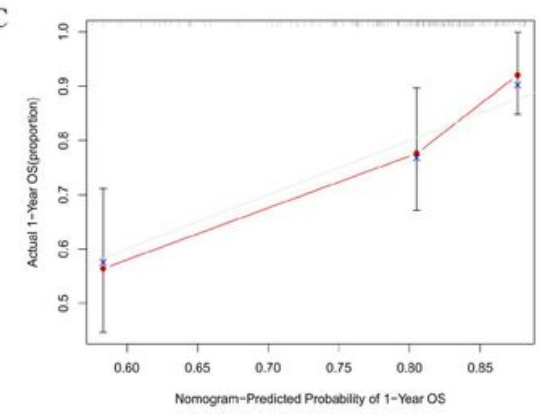

B

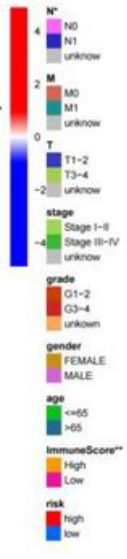

D

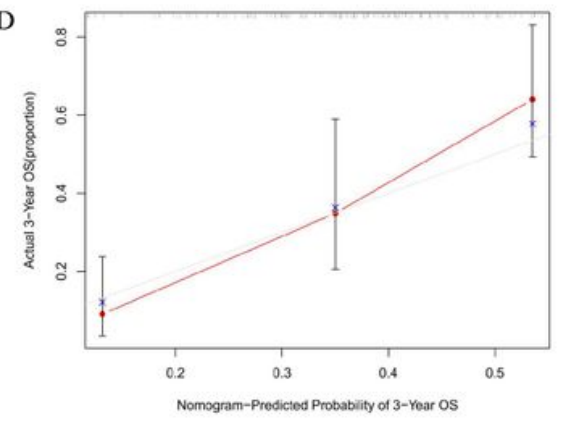

risk $\cdots$
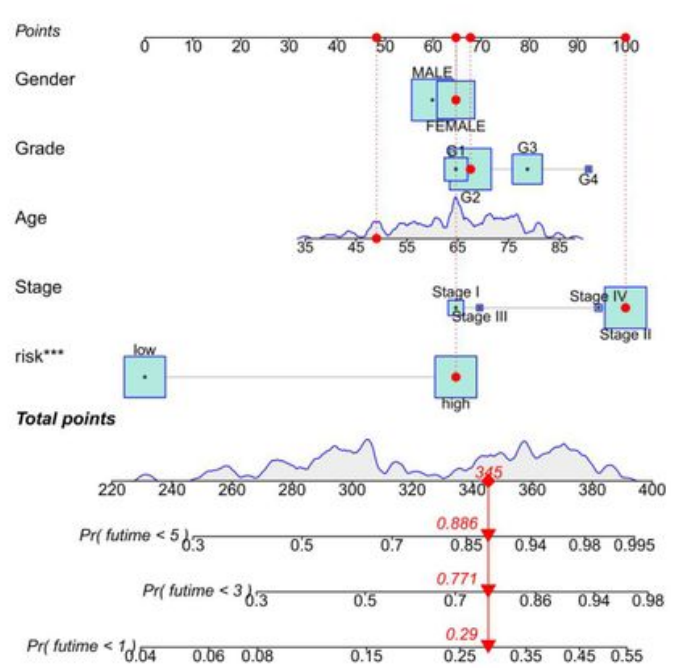

E

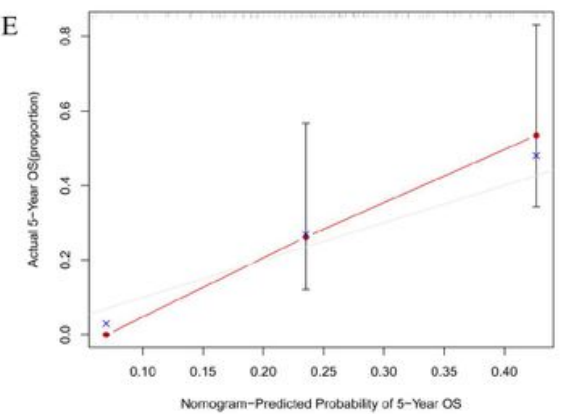

Figure 3

Establishment and verification of the overall survival nomogram for pancreatic cancer patients. (A) The relationship between risk score and clinical symptoms of pancreatic cancer patients. (B) The nomogram consists of clinical characteristics and prognostic signature. (C-E) The nomogram calibration curves were used to predict 1,3 , and 5 years survival rates $\left({ }^{\star} p<0.05 ; * \star p<0.01\right)$. 
A
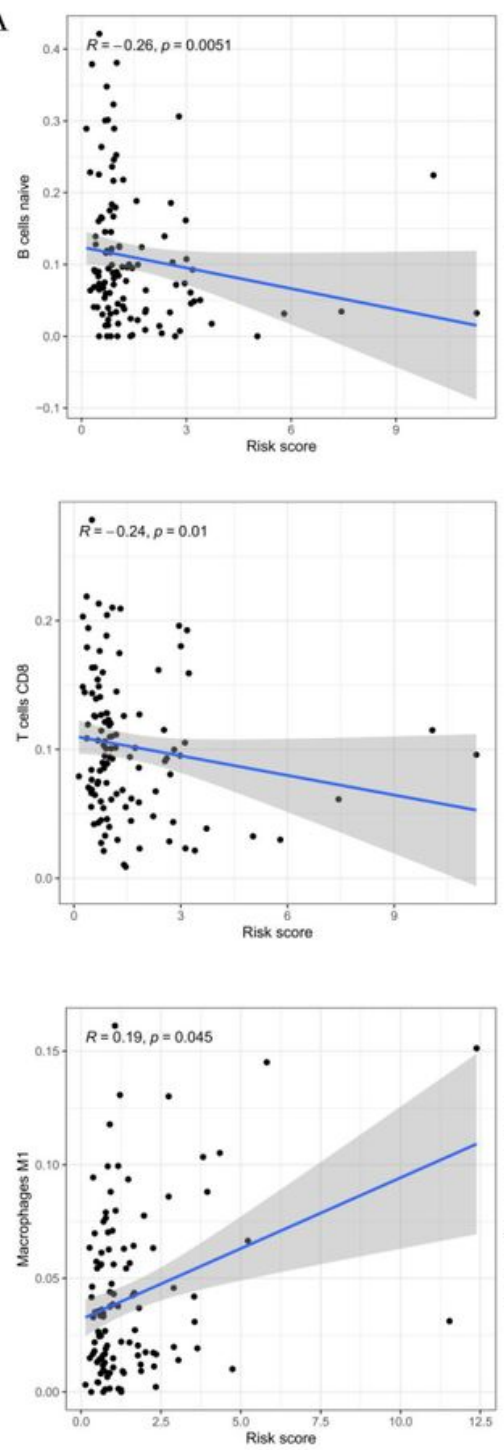
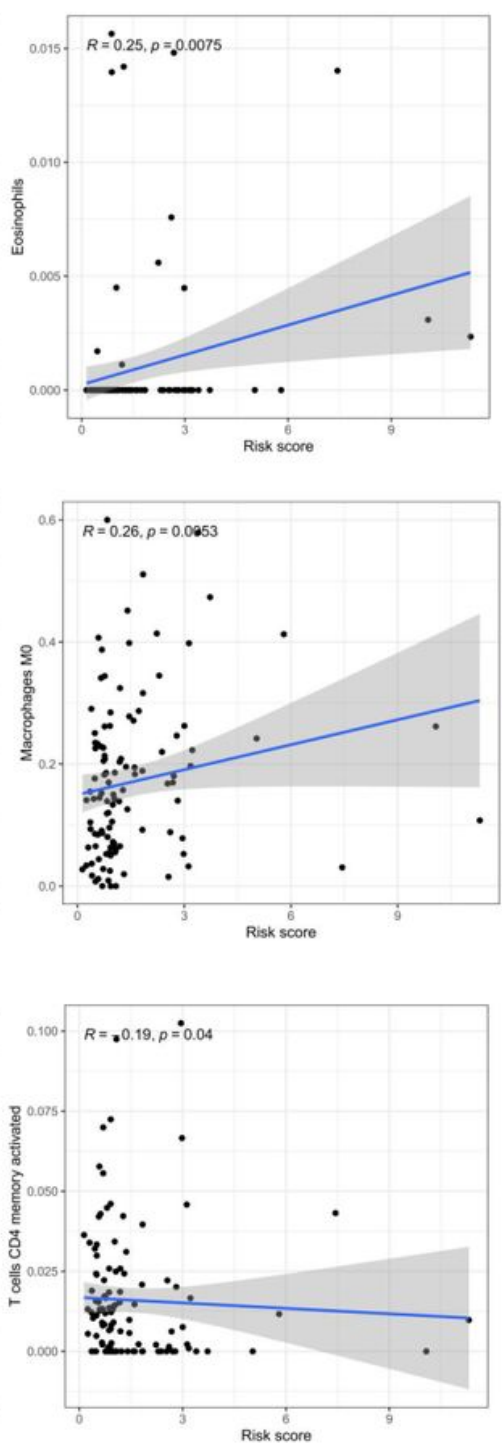

B

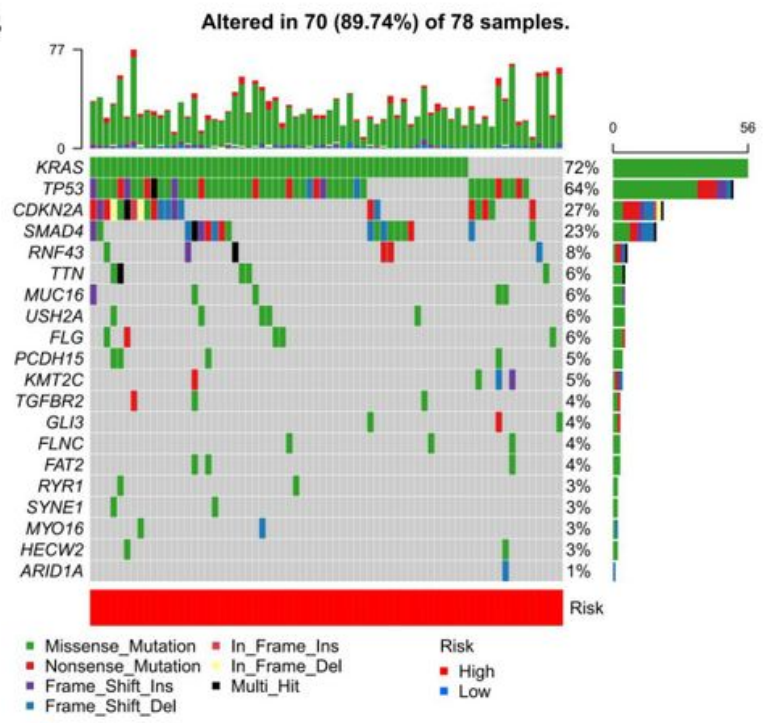

$\mathrm{C}$

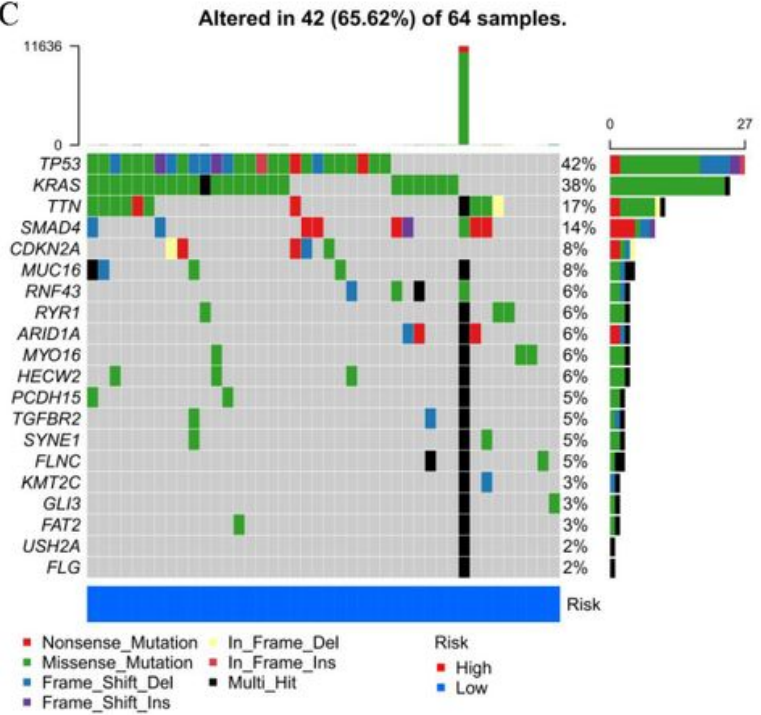

Figure 4

The correlation of the risk score with the immune cells and tumor mutation burden. (A) The correlation between the risk score and immune cells. (B-C) The TMB of PC patients in the high-risk and low-risk groups. 
A

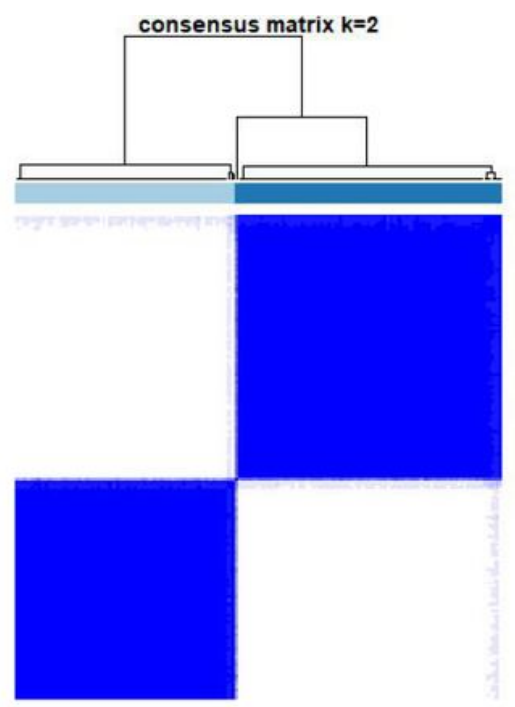

C

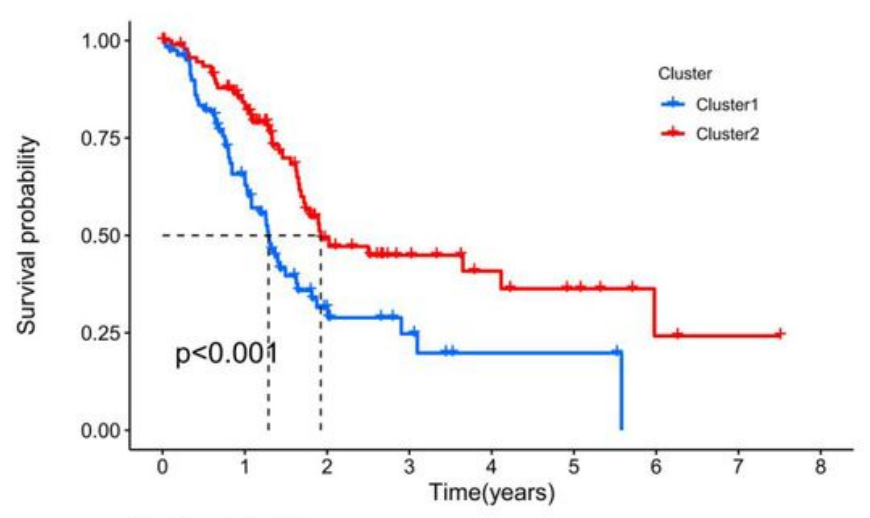

Number at risk

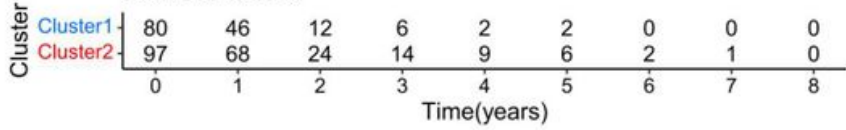

B

- cluster1 - cluster2

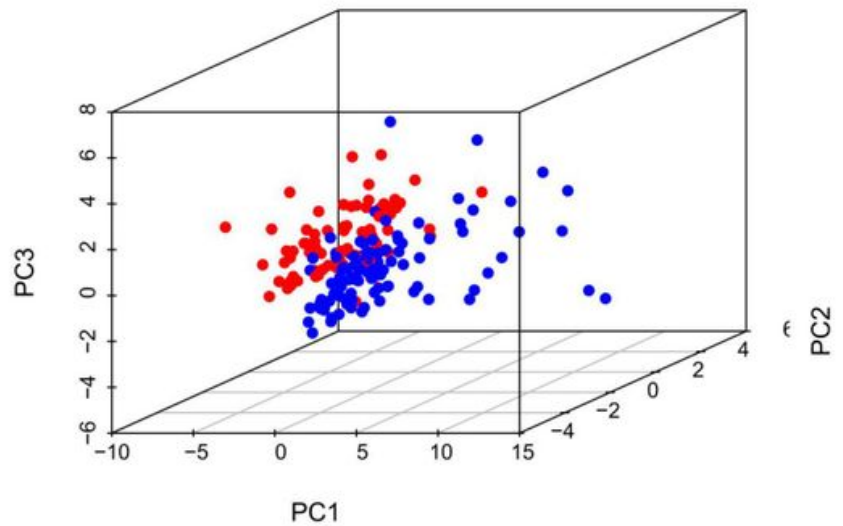

D

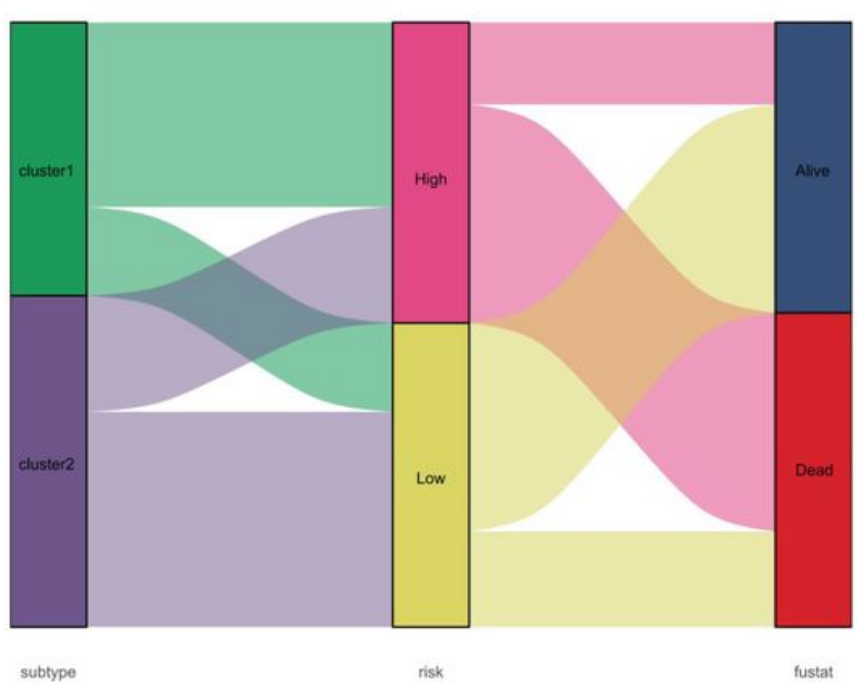

Figure 5

Identification of potential hypoxia subtypes of pancreatic cancer. (A)Hypoxia subtypes of pancreatic cancer were identified when k=2. (B) Principal components analysis between cluster1 and cluster2 on the basis of the hypoxia-related gene sets. (C) Kaplan-Meier survival analysis between cluster1 and cluster2. (D) The correlation of the risk score with Hypoxia subtypes. 
A

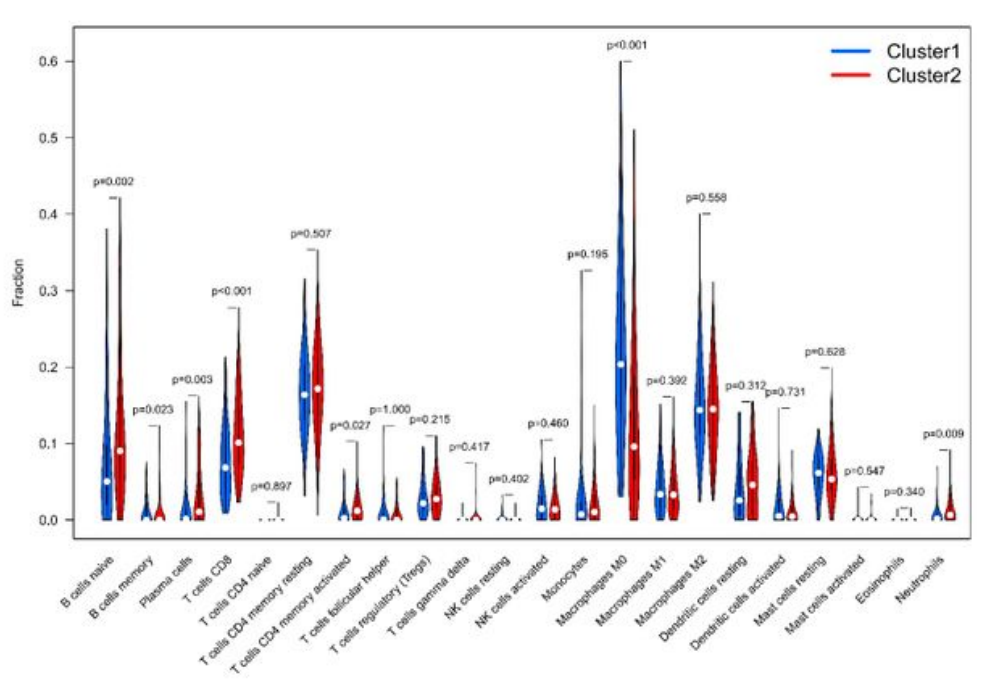

$\mathrm{C}$

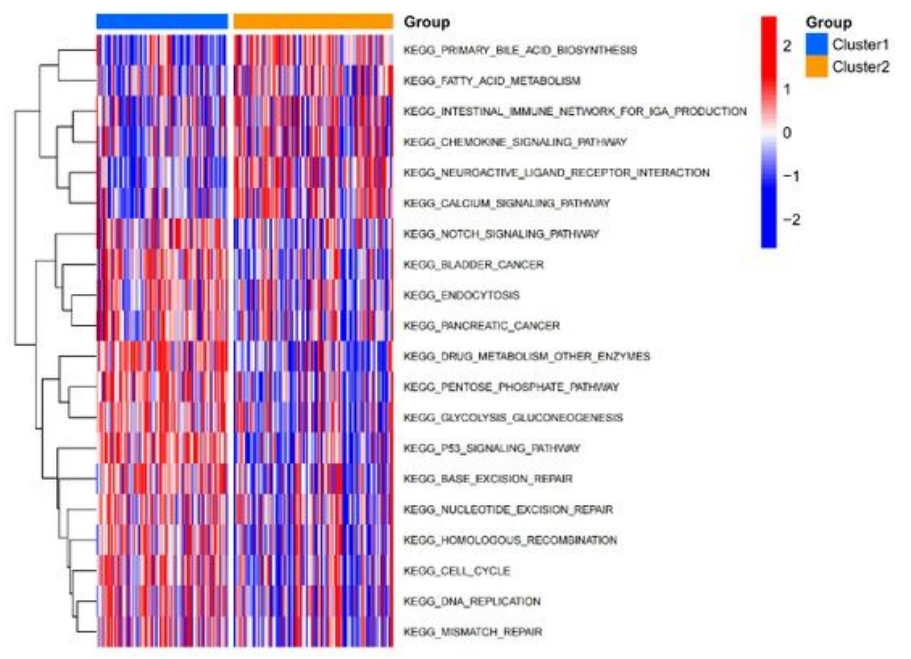

B
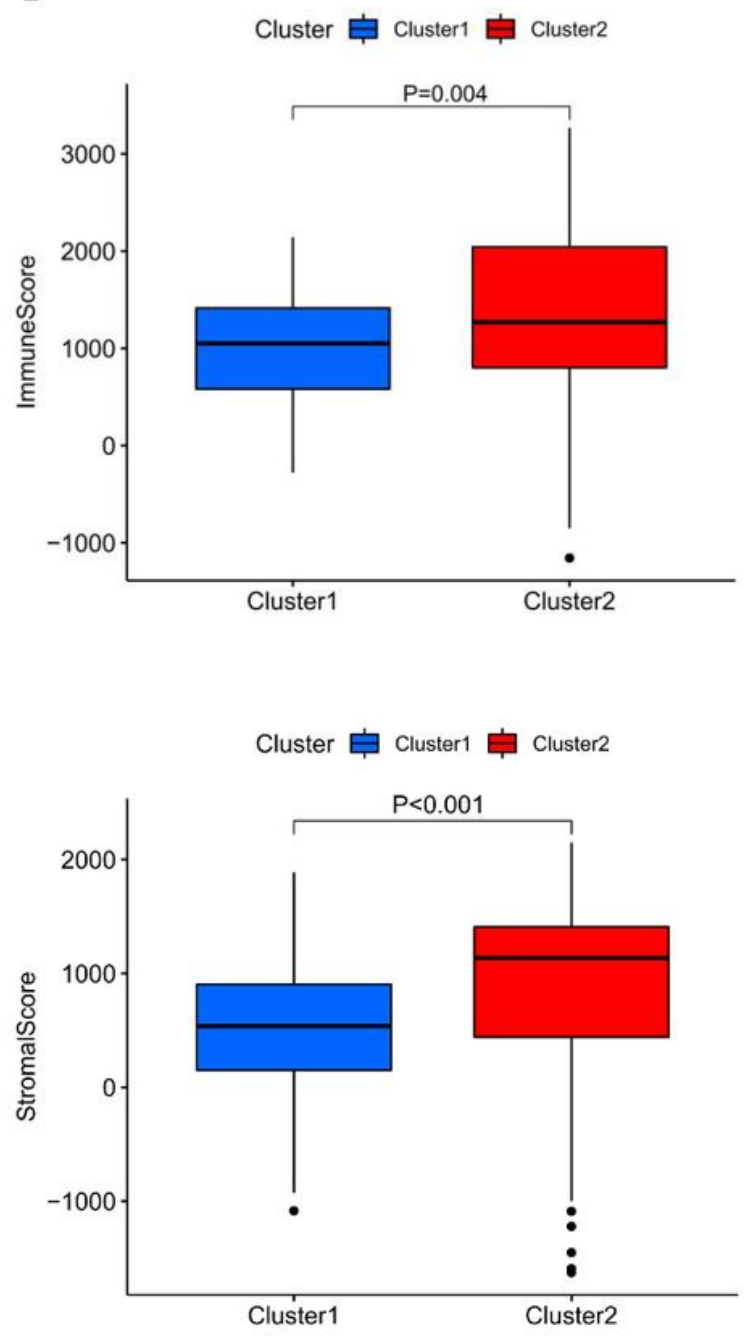

Figure 6

Immune and functional analysis between hypoxia subtypes. (A) The difference of immune cell infiltration between cluster 1 and cluster2. (B) The immune score and stromal score in different hypoxia subtypes. (C) GSVA enrichment analysis showing the activation states of biological pathways in distinct hypoxia subtypes. The heatmap was used to visualize these biological processes. 
A

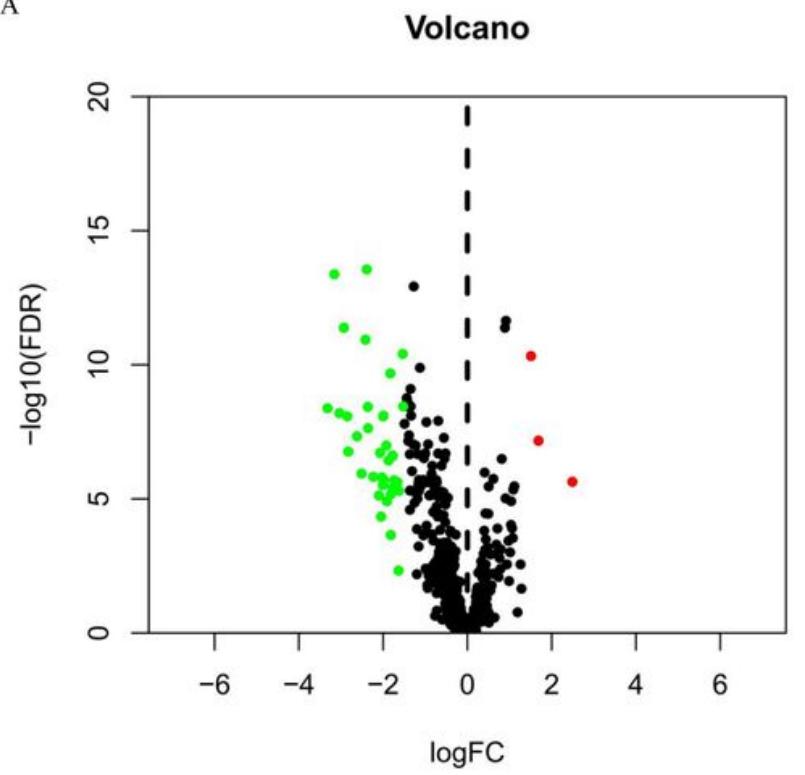

B

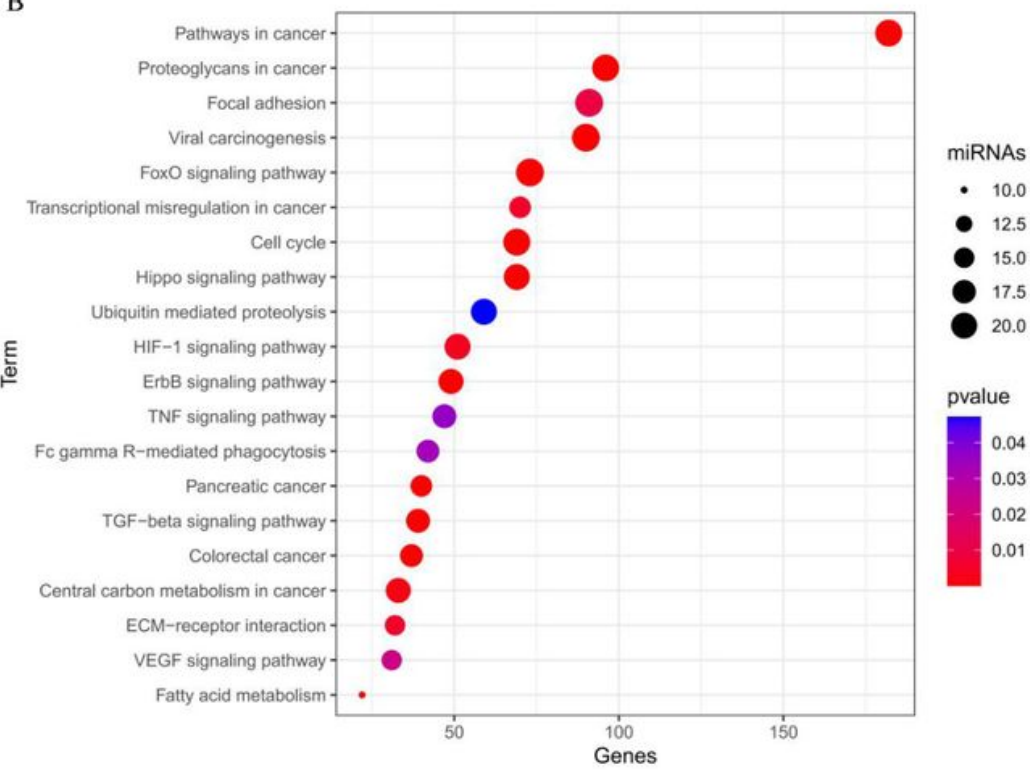

Figure 7

Differentially Expressed MicroRNAs and Enrichment Analysis. (A) Volcano plot of differentially expressed miRNAs. Each red dot represents an upregulated miRNA, and each green dot represents a downregulated miRNA. (B) Kyoto Encyclopedia of Genes and Genomes (KEGG) pathway enrichment analysis of differentially expressed miRNAs. 
A

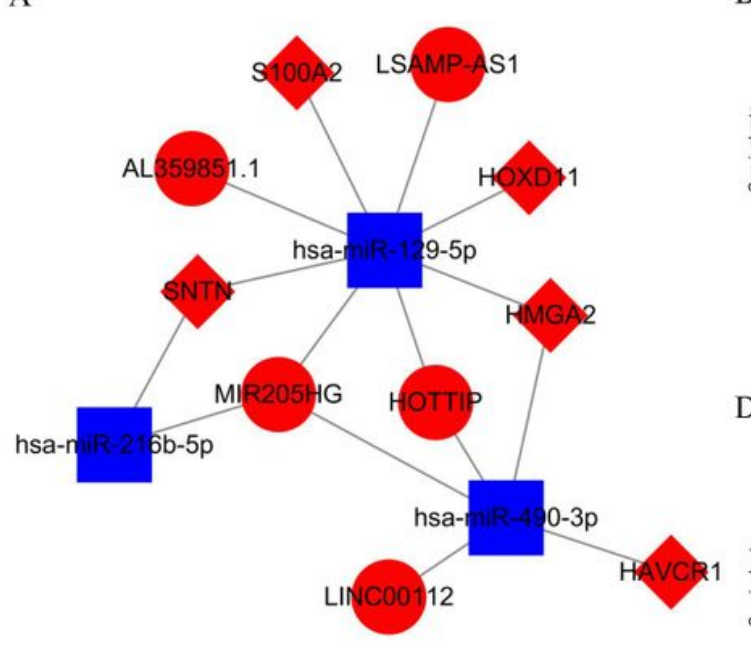

G

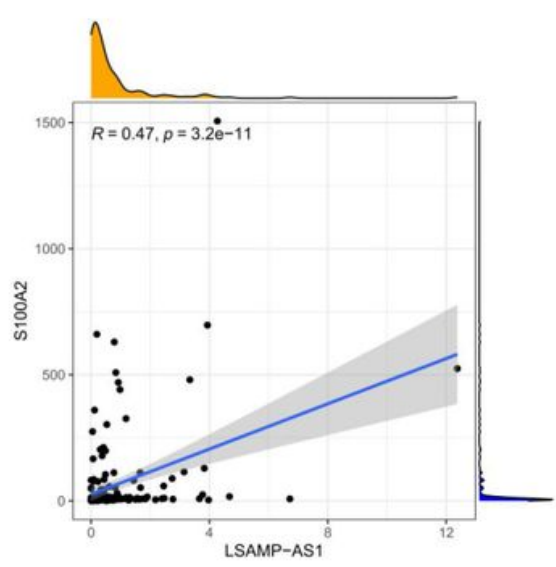

Survival curve $(p=3.467 e-02)$

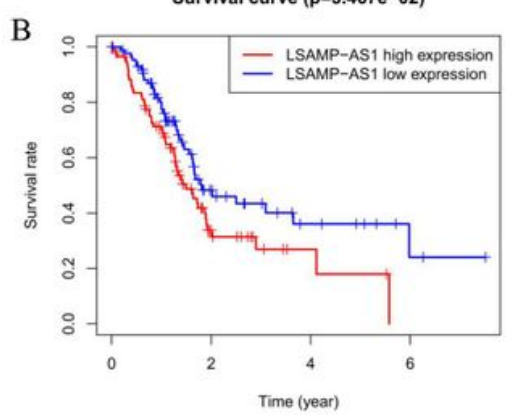

D

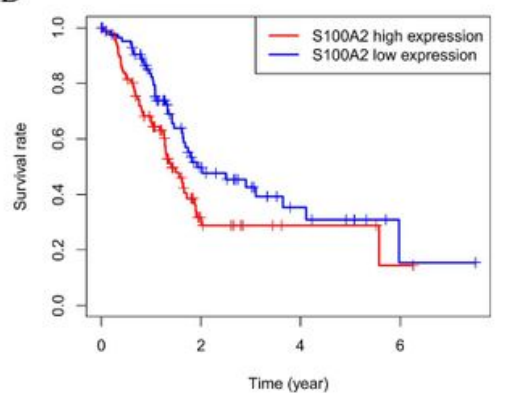

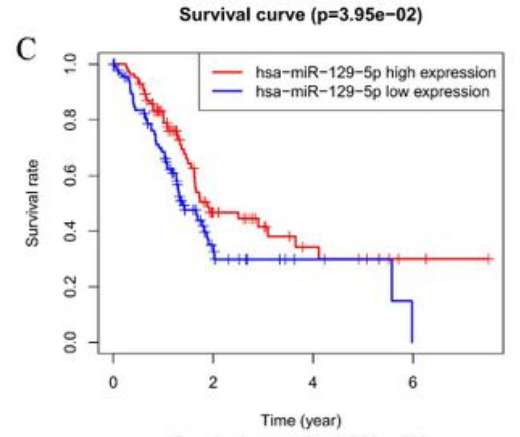

E

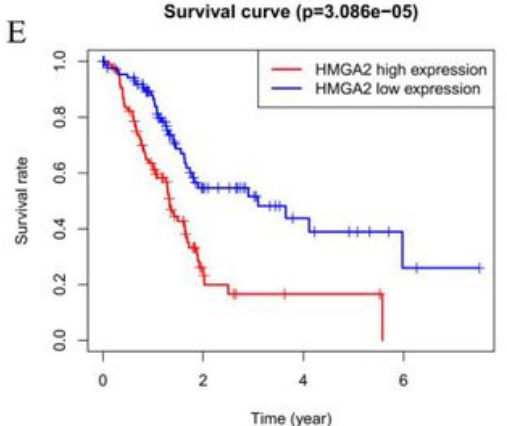

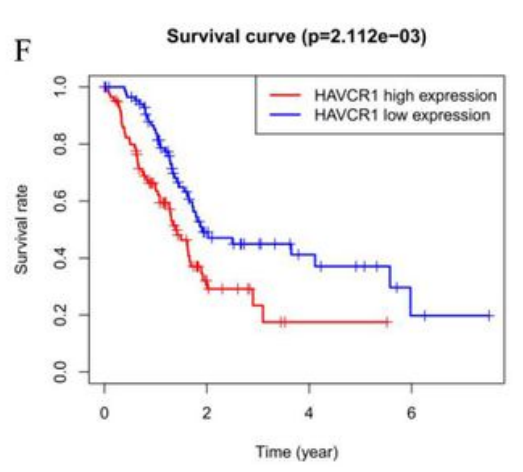

Figure 8

Establishment of a ceRNA regulation network associated with hypoxia. (A) Construction of IncRNAmiRNA-mRNA regulatory networks.(B-F) Kaplan-Meier survival analysis of genes in ceRNA network. (G-H) The correlation between LSAMP-AS1 and S100A2. 
A

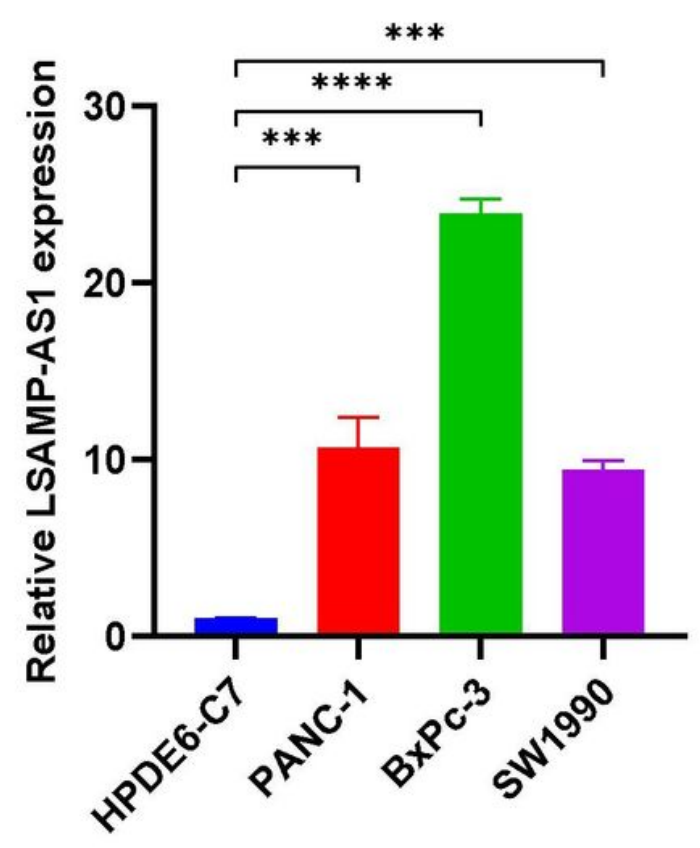

C

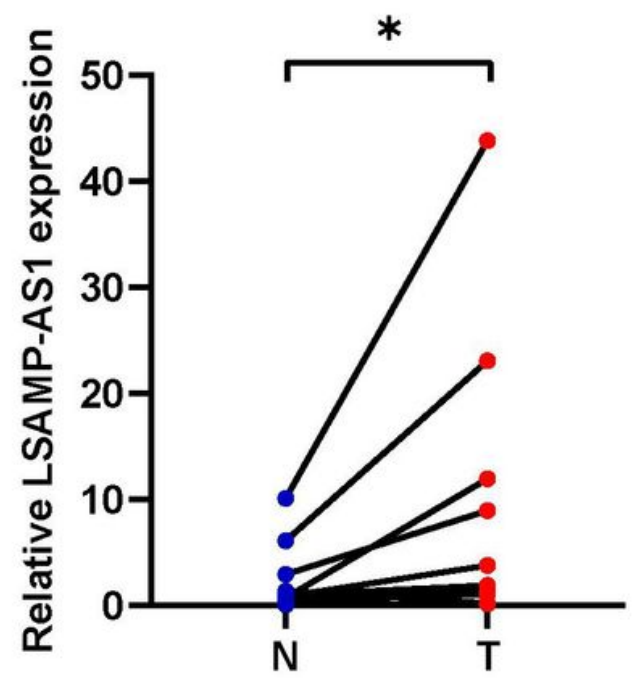

B

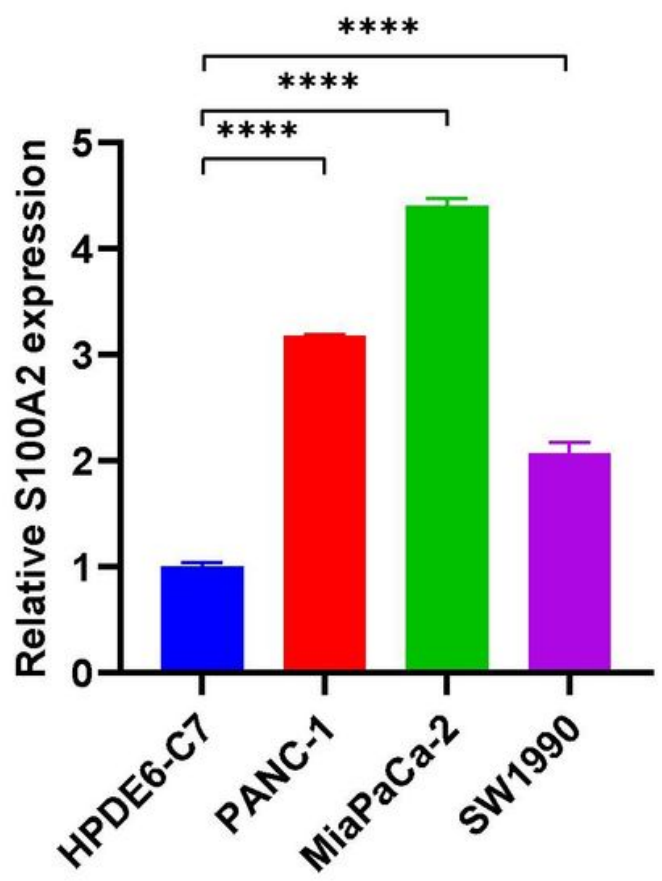

D

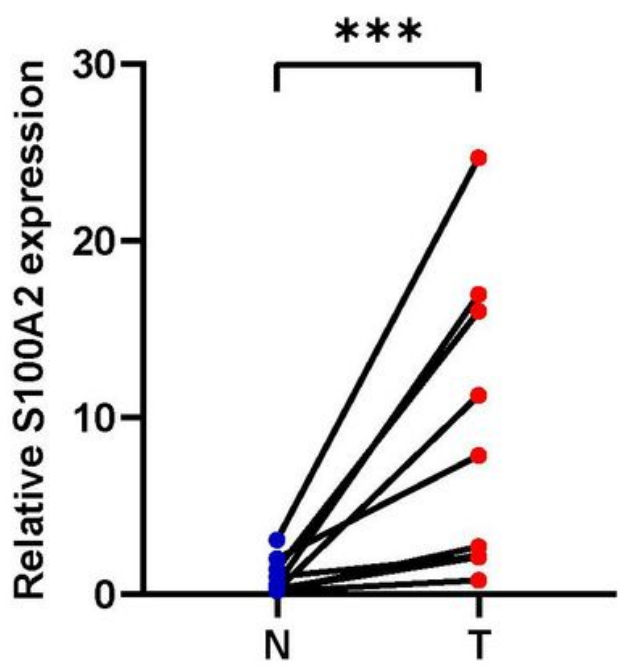

\section{Figure 9}

Relative expression levels of crucial IncRNA and mRNA detected by qRT-PCR. (A) The expression levels of LSAMP-AS1 in PC cells. (B) The expression levels of S100A2 in PC cells. (C) The expression levels of LSAMP-AS1 between normal and PC tissues. (D) The expression levels of S100A2 between normal and PC tissues. ${ }^{*} p<0.05,{ }^{* *} p<0.01$ and ${ }^{* \star *} p<0$.001. IncRNA, long noncoding RNA; mRNA, messenger RNA; qRT-PCR, quantitative real time polymerase chain reaction. 
A Protein expression of $\mathrm{S} 100 \mathrm{~A} 2$ in normal tissues

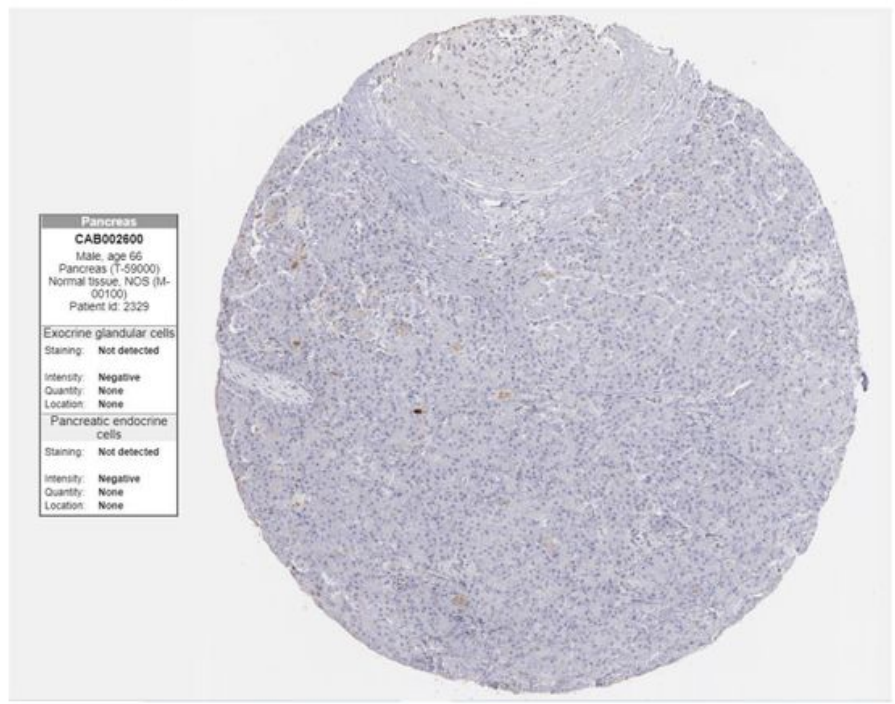

B Protein expression of $\mathrm{S} 100 \mathrm{~A} 2$ in tumor tissues

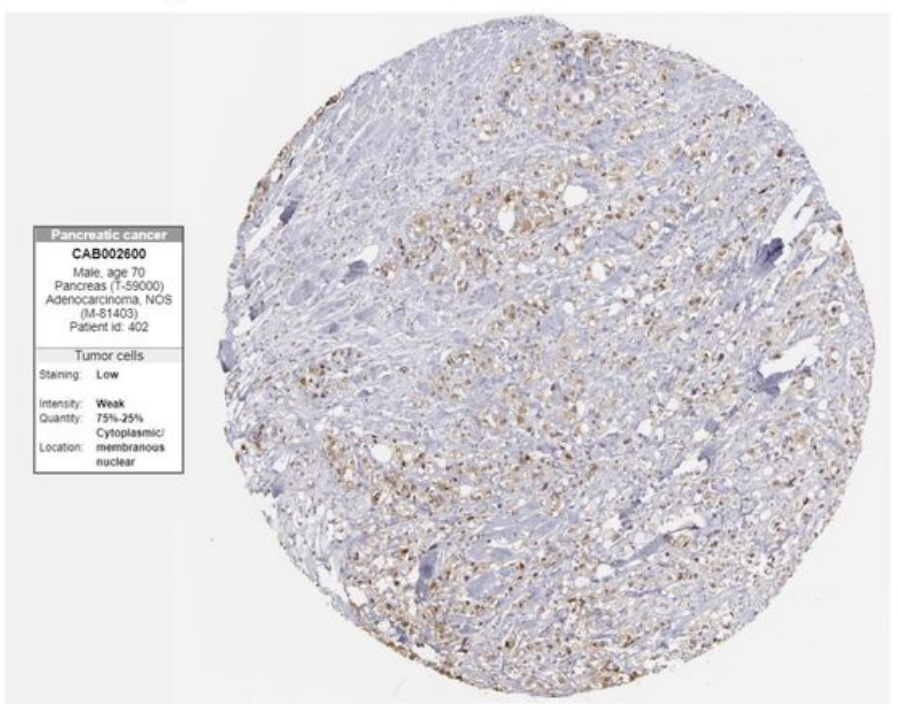

\section{Figure 10}

$\mathrm{IHC}$ of key gene in normal and PC tissues from HPA database. (A) Protein expression of S100A2 in normal pancreatic tissues. (B) Protein expression of S100A2 in PC tissues.

\section{Supplementary Files}

This is a list of supplementary files associated with this preprint. Click to download.

- SupplementaryMaterial.docx 